\title{
A generalization Credibility Premium Estimator with Dependent Risk Structure under the Exponential Premium Principle
}

\author{
Qiang Zhang ${ }^{1}$, Lijun $\mathrm{Wu}^{2}$, Juan Zhang ${ }^{3}$ \\ ${ }^{1)}$ College of Sciences, Shihezi University, Shihezi, Xinjiang, China (zhangqiang189219@163.com) \\ ${ }^{2)}$ College of Mathematics and System Science, Xinjiang University, Wulumuqi, Xinjiang, China (xjmath@edu.cn) \\ ${ }^{3)}$ School of economics, Langfang Teachers College, Langfang, Hebei, China (zhangjuan@163.com)
}

\begin{abstract}
In classical credibility models, claims are assumed to be independent and identically distributed. In many practical situations, however, claims are not identically distributed. In this paper, we present the assumptions of risk heterogeneous portfolio, the credibility models with dependent risk structure have been built under exponential principle. By means of the orthogonal projection method, the credibility estimator is obtained. The results generalize some well known existing results in credibility theory.
\end{abstract}

Keywords — risk heterogeneous, credibility estimator, orthogonal projection, dependent risk

\section{指数保费原理下具有风险相依结构的 广义信度保费估计}

\author{
张强 ${ }^{1} \quad$ 吴黎军 $^{2} \quad$ 张娟 $^{3}$ \\ 1) 石河子大学理学院, 石河子, 新疆, 中国 \\ 2) 新疆大学数学与系统科学学院, 乌鲁木齐, 新疆, 中国 \\ 3) 廊坊师范学院经济学院, 廊坊, 河北, 中国
}

摘 要 在经典的信度模型中, 假设索赔时独立同分布的。然而, 在实务中, 索赔不一定满足同分布的假设。提出风险异质保单 的假设, 在指数保费原理下建立了具有风险间相依结构的信度模型。采用正交投影的方法, 得到了指数保费原理下具有风险间相依结 构信度估。这一结果推广了经典的信度模型。

关键词 风险异质，信度估计，正交投影，风险相依

1. 引言

在非寿险精算中，信度理论可以用来有效地估计未来 保费, 其方法是将制定的保费表示为投保人个人的索赔经 历与先验保费加权和。关于经典的信度模型, 详细可见[1], 通常得到的保费估计都是纯保费，但在实务中，保险公司 为获取收益甚至避免破产, 收取的保费须具有正的安全负 荷, 而不单纯是纯保费。文献 ${ }^{[2]}$ 在指数加权损失函数下得 到了 Esscher 保费的信度估计。文献 ${ }^{[3]}$ 修正了 [2] 的结果,

国家自然科学基金项目支持（资助号：207-60396）
并给出了 Esscher 保费估计的相合性证明。指数保费原理是 精算学中最为重要的保费原理之一, 是除期望值原理之外 满足最多优良性质的保费原理, 而且形式上简单。文献 ${ }^{[4]}$ 在平方损失函数下建立了指数保费原理下的信度模型, 并 多数学者在利用保单组合来预测未来索赔 $X_{i n_{i}+1}$ 时, 通常假 设 $K$ 个保单风险间是相互独立的。然而, 风险之间的独立 性假设仅是实际风险的一种理想化近似, 大多情况下风险 之间存在着某种相关。文献 ${ }^{[5]}$ 在提出一种共同效应随机变 量的前提下, 建立了风险间有某种相依结构的信度模型。 文献[6]提出了共同效应相依的信度模型, 推广了 [5]的结 
果。文献 ${ }^{[7]}$ 在风险等相关的条件下, 得到了多合同模型的 估计。文献 ${ }^{[8]}$ 讨论了具有风险相依结构的 Bühlmann 信度模 型。本文考虑风险具有某种相依结构, 在风险异质保单下, 引入平方损失函数, 并采用正交投影的方法求解最优化问 题, 得到了目标问题的最优解, 从而在指数保费原理下得 到了具有风险间相依结构的广义信度公式

\section{2. 预备知识与模型假设}

若随机变量 $X$ 满足 $M(t)=E[\exp (t X)]<\infty, t \in R$, 则 称 $X$ 为损失随机变量。

定义 1 损失随机变量 $X$ 的指数保费原理下的保费为

$$
H(X, \Theta)=\frac{1}{\alpha} \log [E(\exp (\alpha X) \mid \Theta)] \quad \alpha>0
$$

由于风险参数 $\Theta$ 在保险实务中一般是未知的, 通常是 要用历史索赔数据给出 $\Theta$ 的估计。本文考虑保单组合来给 出 $H(X, \Theta)$ 的一致估计, 假设有 $K$ 个保单组合, 其中 $X_{i j}$ 表 示第 $i$ 个投单第 $j$ 个时期的历史索赔数据, 在这里 $i=1,2, \cdots, K j=1,2, \cdots, n_{i}$, 在实务中 $n_{i}$ 对于不同的 $i$ 一般是 不相同的。历史索赔数据可表示为 $\boldsymbol{X}=\left(\boldsymbol{X}_{1}^{\prime}, \boldsymbol{X}_{2}^{\prime}, \cdots \boldsymbol{X}_{K}^{\prime}\right)^{\prime}$, 其 中 $\boldsymbol{X}_{i}=\left(X_{i 1}, X_{i 2}, \cdots X_{i n_{i}}\right)^{\prime}$ 。类似于文献[1], 第 $i$ 个投保个 体未来索赔 $X_{i n_{i}+1}$ 基于样本函数类 $L(X, 1)=\left\{c_{0}+\sum_{i=1}^{K} c_{i}^{\prime} X_{i}\right.$, $\left.c_{0} \in R, c_{i} \in R^{n_{i}}\right\}$ 得到的。本文给出的模型假设如下

假设 1 在给定风险参数 $\Theta_{i}=\theta_{i}$ 下, 随机变量 $X_{i j}$, $j=1,2, \cdots n_{i}$ 是相互独立的, 且具有条件 $E\left(e^{\alpha X_{\ddot{i}}} \mid \Theta_{i}\right)=\beta_{j} \mu\left(\Theta_{i}\right)$ 和 $\operatorname{Var}\left(e^{\alpha X_{\ddot{i}}} \mid \Theta_{i}\right)=\tau_{j}\left(\Theta_{i}\right)+\varphi_{j} v\left(\Theta_{i}\right)$, $i=1,2, \cdots, K, j=1,2, \cdots, n_{i}$ 。

假设 2 记 $\boldsymbol{\Theta}=\left(\Theta_{1}, \cdots, \Theta_{K}\right)^{\prime}, \Theta$ 具有相同的结构分布函 数 $\pi(\theta)$, 且在给定 $\Theta$ 下, 随机变量 $X_{1}, X_{2}, \cdots X_{K}$ 相互独立。

假设 3 风险 $\Theta_{1}, \cdots, \Theta_{K}$ 之间存在相依性, 用相关矩阵来 刻画, 有 $\operatorname{Cov}\left(\mu\left(\Theta_{i}\right), \mu\left(\Theta_{j}\right)\right)=\rho_{i} \rho_{j}$, 其中 $i \neq j, i, j=1,2$, $\cdots, K$ 。引入下面的记号

$$
\mathrm{E}\left(\mu\left(\Theta_{i}\right)\right)=\mu_{i}, \quad \mathrm{E}\left(v\left(\Theta_{i}\right)\right)=v, \quad \mathrm{E}\left(\tau_{j}\left(\Theta_{i}\right)\right)=\tau_{j} 。
$$

以下是要用到的引理。

引理 1 随机变量 $Y$ 在线性空间 $L(X, 1)$ 上的正交投影 称为非齐次信度估计, 即

$$
\operatorname{proj}(Y \mid L(X, 1))=E(Y)+\sum_{Y X} \sum_{X X}^{-1}(X-E(X))
$$

其中 $\sum_{Y X}$ 是 $Y$ 与 $X$ 的协方差矩阵, $X=\left(X_{1}^{\prime}, X_{2}^{\prime}, \cdots X_{K}^{\prime}\right)^{\prime}$ 。 证明见 ${ }^{[1]}$ 。

引理 2 设 $A, B, C, D$ 为具有适当阶数的矩阵, 则有下 面的求逆公式, 证明可见 ${ }^{[9]}$ 。

$$
(A+B C D)^{-1}=A^{-1}-A^{-1} B\left(C^{-1}+D A^{-1} B\right)^{-1} D A^{-1}
$$

\section{3. 信度保费估计}

本文的目标在历史索赔数据 $\boldsymbol{X}=\left(\boldsymbol{X}_{1}^{\prime}, \boldsymbol{X}_{2}^{\prime}, \cdots \boldsymbol{X}_{K}^{\prime}\right)^{\prime}$ 下, 通 过求解最小化问题

$$
\min _{c_{0}, c_{i j} \in R} E\left[\left(e^{\alpha X_{i, n+1}}-c_{0}-\sum_{s=1}^{K} \sum_{j=1}^{n_{i}} c_{i j} e^{\alpha X_{i j}}\right)^{2}\right]
$$

来预测指数保费

$$
H\left(X, \Theta_{i}\right)=\frac{1}{\alpha} \log \left[E\left(e^{\alpha X_{i, n_{i}+1}} \mid \Theta_{i}\right)\right]
$$

为方便表示, 引入 $Y_{i j}=\exp \left(\alpha X_{i j}\right)$, 和 $\boldsymbol{Y}=\left(\boldsymbol{Y}_{1}^{\prime}, \cdots \boldsymbol{Y}_{K}^{\prime}\right)^{\prime}$, 其中 $\boldsymbol{Y}_{i}=\left(Y_{i 1}, Y_{i 2}, \cdots Y_{i n_{i}}\right)^{\prime}$, 此时有

$$
\min _{c_{0} \in R, c_{s} \in \boldsymbol{R}^{n_{s}}} E\left[\left(Y_{i, n_{i}+1}-c_{0}-\sum_{s=1}^{K} \boldsymbol{c}_{s}^{\prime} \boldsymbol{Y}_{s}\right)^{2}\right]
$$

得到的估计记作 $H\left(X, \Theta_{i}\right)^{*}$ 。根据引理 1 和 2 , 很容易得到 $H\left(X, \Theta_{i}\right)^{*}$ 。首先给出下面的定理, 为表示方便引入以下记 号

$$
t_{j}=\frac{\beta_{j}^{2}}{\tau_{j}+\varphi_{j} v}, \quad m_{i}=\sum_{j=1}^{n_{i}} t_{j}, \quad \lambda_{i}=\rho_{i}^{2} m_{i}, \quad \lambda=\sum_{i=1}^{K} \lambda_{i}, \quad s_{i}=\sum_{j=1}^{n_{i}} \frac{t_{j}}{\beta_{j}}
$$

个体均值和总体均值分别为

$$
\bar{Y}_{i}=\frac{1}{s_{i}} \sum_{j=1}^{n_{i}} \frac{t_{j} e^{\alpha X_{i j}}}{\beta_{j}} \text { 和 } \overline{\bar{Y}}_{s}=\frac{1}{\sum_{i=1}^{K} \rho_{i} s_{i}} \sum_{i=1}^{K} \bar{Y}_{i} \rho_{i} s_{i} 。
$$

定理 1 在假设 1-3 下, 第 $i$ 个合同在指数保费原理下 的信度保费为

$$
H\left(X, \Theta_{i}\right)^{*}=\frac{1}{\alpha} \log \left\{\beta_{n_{i}+1}\left[(1-Z) \mu+Z \overline{\bar{Y}}_{s}\right]\right\}
$$

其中 $Z=\frac{\beta_{n_{i}+1} \rho_{i} \sum_{i=1}^{K} \rho_{i} s_{i}}{1+\lambda}$ 称为信度因子。

$$
\text { 证明记 } \boldsymbol{\Theta}=\left(\Theta_{1}, \cdots, \Theta_{K}\right)^{\prime} \text { 和 } \boldsymbol{\mu}(\boldsymbol{\Theta})=\left(\mu\left(\Theta_{1}\right), \cdots, \mu\left(\Theta_{K}\right)\right)^{\prime}
$$

则有

$$
E\left(Y_{i i_{i}+1}\right)=\beta_{n_{i}+1} \mu
$$

由于

和

$$
\left(E_{i} Y=E\left[Y \mid \Theta_{i}\right)\right]=\boldsymbol{T}_{i} \mu
$$

$$
E(\boldsymbol{Y})=E\left(\boldsymbol{Y}_{1}^{\prime}, \cdots, \boldsymbol{Y}_{K}^{\prime}\right)^{\prime}=\left(\boldsymbol{T}_{1}^{\prime} \cdots \boldsymbol{T}_{K}^{\prime}\right)^{\prime} \mu
$$

运用方差的条件期望公式有

$$
\begin{aligned}
\sum_{Y_{i, n_{i}+1} \mathbf{Y}} & =E\left[\operatorname{Cov}\left(Y_{i, n_{i}+1} \boldsymbol{Y} \mid \boldsymbol{\Theta}\right)\right]+\operatorname{Cov}\left[E\left(Y_{i, n_{i}+1} \mid \boldsymbol{\Theta}\right), E(\boldsymbol{Y} \mid \boldsymbol{\Theta})\right] \\
& =\beta_{n_{i}+1} \rho_{i}\left(\boldsymbol{T}_{1}^{\prime} \rho_{1}, \cdots, \boldsymbol{T}_{K}^{\prime} \rho_{K}\right)
\end{aligned}
$$


通过简单计算可得 $\sum_{Y Y}$ 为

$$
\begin{aligned}
\sum_{\boldsymbol{Y Y}}= & \operatorname{diag}\left(\Lambda_{1}, \cdots, \Lambda_{K}\right) \\
& +\left(\boldsymbol{T}_{1} \rho_{1}, \cdots, \boldsymbol{T}_{K} \rho_{K}\right)^{\prime}\left(\boldsymbol{T}_{1}^{\prime} \rho_{1}, \cdots, \boldsymbol{T}_{K}^{\prime} \rho_{K}\right)
\end{aligned}
$$

其中 $\Lambda_{i}=\operatorname{diag}\left(\tau_{1}+\varphi_{1} v, \cdots, \tau_{n_{i}}+\varphi_{n_{i}} v\right)$ 。

由引理 2 矩阵求逆公式, 可得

$$
\begin{aligned}
\sum_{Y Y}^{-1}=\operatorname{diag}\left(\Lambda_{1}^{-1}, \cdots, \Lambda_{K}^{-1}\right)-\frac{1}{1+\lambda} & \\
& \left.P\left(Q \cdots, \beta_{K} Q^{\prime}\right) \kappa_{1} Q \cdots, \rho_{K} Q_{K}\right)
\end{aligned}
$$

其中 $Q_{i}=\left(\frac{\beta_{1}}{\tau_{1}+\varphi_{1} v}, \cdots, \frac{\beta_{n_{i}}}{\tau_{n_{i}}+\varphi_{n_{i}} v}\right)$ 。

由引理 1 , 结合(6)及(11)可得信度估计 $\hat{Y}_{i, n_{i+1}}^{*}$ 为

$$
\begin{aligned}
\hat{Y}_{\dot{i n}_{i}+1}^{*}= & E\left(Y_{\dot{t i n}_{i}+1}\right)+\sum_{Y_{\dot{t i}_{i}+1} Y} \sum_{Y Y}^{-1}(\boldsymbol{Y}-E(\boldsymbol{Y})) \\
= & \beta_{n_{i}+1} \mu_{i}+\frac{1}{1+\lambda} \beta_{n_{i}+1} \rho_{i} \sum_{i=1}^{K} \rho_{i} \sum_{j=1}^{n_{i}} \frac{t_{j} Y_{i j}}{\beta_{j}} \\
& -\frac{1}{1+\lambda} \beta_{n_{i}+1} \rho_{i} \sum_{i=1}^{K} \rho_{i} \sum_{j=1}^{n_{i}} \frac{t_{j} \mu}{\beta_{j}} \\
= & \beta_{n_{i}+1} \mu+\frac{1}{1+\lambda} \beta_{n_{i}+1} \rho_{i} \sum_{i=1}^{K} \rho_{i} s_{i} \overline{\bar{Y}}_{s}-\frac{1}{1+\lambda} \beta_{n_{i}+1} \rho_{i} \sum_{i=1}^{K} \rho_{i} s_{i} \mu \\
= & \beta_{n_{i}+1}\left[Z \overline{\bar{Y}}_{s}(1-Z) \mu\right]
\end{aligned}
$$

故在指数保费原理下的第 $i$ 个合同的非齐次信度估计为

$$
H\left(X, \Theta_{i}\right)^{*}=\frac{1}{\alpha} \log \left\{\beta_{n_{i}+1}\left[(1-Z) \mu+Z \overline{\bar{Y}}_{s}\right]\right\}
$$

注在定理 1 中, 若有 $\beta_{j}=1, j=1,2, \cdots, n_{i}$ 且

$$
\operatorname{Cov}\left(\mu\left(\Theta_{i}\right), \mu\left(\Theta_{j}\right)\right)= \begin{cases}0, & i \neq j \\ \tau_{0}^{2}, i=j\end{cases}
$$

和

$$
\tau_{1}+\varphi_{1} v=\cdots=\tau_{n_{i}}+\varphi_{n_{i}} v=\sigma_{0}^{2}, \quad i=1, \cdots, K
$$

信度估计

$$
H\left(X, \Theta_{i}\right)^{*}=\frac{1}{\alpha} \log \left\{\left[\left(\frac{\sigma_{0}^{2}}{n_{i} \tau_{0}^{2}+\sigma_{0}^{2}}\right) \mu+\frac{n_{i} \tau_{0}^{2}}{n_{i} \tau_{0}^{2}+\sigma_{0}^{2}} \bar{u}_{i}\right]\right\}
$$

其中 $\bar{u}_{i}=\frac{1}{n_{i}} \sum_{j=1}^{n_{i}} e^{\alpha X_{i j}}$, 见文献[4], 定理 1 是其的一个推广。

\section{4. 结论}

本文在风险异质保单下, 利用相关矩阵表示现实中风 险相依结构, 将目标保费估计限定在经验数据的线性函数 类上, 建立了最优问题, 并采用正交投影的方法求解最优 化问题, 得到了目标问题的最优解, 从而在指数保费原理 下得到了具有风险间相依结构的广义信度公式。结果表明, 所得的公式具有经典的信度估计形式, 打破了风险同质保 单的限制, 推广了信度估计的应用范围, 为保险公司厘定 未来保费提供了参考依据。此外, 对于公式中相关参数的 需要用统计学知识来估计, 如何得到无偏估计及相合估计 是一个需进一步研究的问题。

\section{参考文献(References)}

[1] H. Bühlmann, A. Gialer. A course in credibility theory and its application, Netherlands: Springer, 2005.

[2] H. -U Gerber, A. Arbor. "Credibility for Esscher premium", Mitleilungen der VSVM, vol. 80, no. 3, pp. 307-321, March 1980.

[3] M.-L Pan, R.-M Wang, X.-Y Wu. "On the consistency of credibility premiums regarding Esscher Principle", Insurance: Mathematical and Economics, vol. 42, no. 1, pp. 119-126, February 2008.

[4] L.-M Wen, W.Wang, J.-L Wang. "The credibility Premiums for Exponential Principle", Acta Mathematical Sinica, English Series, vol. 27, no. 11, pp. 2217-2228, Novembor 2011.

[5] K.-L Yeo, E.Valdez. "A claim dependence with common effects in credibility models" Insurance: Mathem atics and Economics, vol.38, no. 3, pp.609-629, June 2006.

[6] L.-M Wen, X.-Y Wu, X. Zhou. "The credibility premiums for models with dependence induced by common effects", Insurance: Mathematics and Economics, vol. 44, no. 1, pp.19-25, February 2009.

[7] L. -M Wen,W.-L D, "The credibility models with equal correlate on risks", J Syst Sci Complex, vol. 24, no. 3, pp.532-539, June 2011.

[8] L.-M Wen, H.-L Gong,J.-L Wang. "The Bühlmann credibility models with dependent risk structure", Acta Mathematicae appl icatae sinica, vol. 33, no. 4, pp. 723-740, July 2010.

[9] R. Rao, H. Toutenbur. Linear Models, New York, Springer, 1999. 